\title{
LHC discoveries and particle physics concepts for education
}

\author{
Farid Ould-Saada ${ }^{1}$ \\ University of Oslo \\ P.O.Box 1048. 0316 Oslo, Norway \\ E-mail: farid.ould-saadalfys.uio.no
}

\begin{abstract}
The LHC data have been successfully deployed in International Masterclasses where the Higgs discovery and other measurements and scientific methods could be shared with high school and other students. The promises of the $13 \mathrm{TeV}$ era in terms of new discoveries and the opportunity offered by the CERN open data portal triggered the extension of the educational material based on real data. After a brief account of the current successes, we describe the newly implemented features and discuss future plans. Research projects based on large LHC data samples open new possibilities for university students and provide a strong support to both the theoretical and experimental lectures. Tools are already available to analyze larger datasets. The invariant mass is successfully mastered to measure properties of known particles and discover new ones. Other achievements include the understanding of the concepts of 'event' and 'statistics'. More concepts need to be brought closer to students. Missing energy-momentum (ETmiss), a key concept closely related to dark matter (DM) and supersymmetry (SUSY), is being further exploited. For example, signals of graviton resonances in di-lepton, di-photon and di-Z boson mass distributions, and the exploitation of ETmiss to study di-lepton invariant mass endpoints of SUSY particles have been implemented. These will contribute to the ambition to tune and expand the educational material to follow the LHC 'heartbeats' and to influence the textbooks and teaching methods already at the level of high schools.
\end{abstract}

The European Physical Society Conference on High Energy Physics 22-29 July 2015

Vienna, Austria

${ }^{1}$ Speaker, On behalf of the International Particle Physics Outreach Group 


\section{Introduction}

The International Particle Physics Outreach Group (IPPOG) has developed an educational activity that brings the excitement of cutting-edge particle physics research into the classroom [1]. Each year, since 2005, thousands of high school students in many countries all over the world come to nearby universities or research centres for one day in order to unravel the mysteries of particle physics and be "scientists for a day" [2]. The LHC data have been successfully deployed since 2010 in International Masterclasses (IMC) where students perform various measurements based on proton-proton, lead-lead and proton-lead collisions. The Higgs discovery, the investigation of the quark-gluon plasma phenomenon and other measurements and scientific methods could be shared with the students $[3][4][5][6][7][8][9][10]$.

The ambition to bring to the "classrooms" important LHC discoveries is realized using the recent discovery of the Higgs boson. The promises of the $13 \mathrm{TeV}$ LHC era in terms of new discoveries and the opportunity offered by the CERN open data portal [11] triggered the extension of the educational materials. Interesting discussions started on how to introduce more concepts should new phenomena be discovered at the LHC.

\section{Bringing Particle Physics to Students at Multiple Levels}

Pre-High School, High School and University students can learn about particle physics in ways that are appropriate to their own level of physics understanding. We identify four levels. The introductory level (see for example [12]) is accessible to most students. It uses limited amounts of data to convey key concepts, such as the importance of conservation laws and the use of indirect evidence, through shorter activities. Students taking part in level 1 analyse one variable; they determine patterns, organize into a table or graphical representation and perform simple calculations, such as the calculation of the mass of the $\mathrm{Z}$ particle (Figure 1).

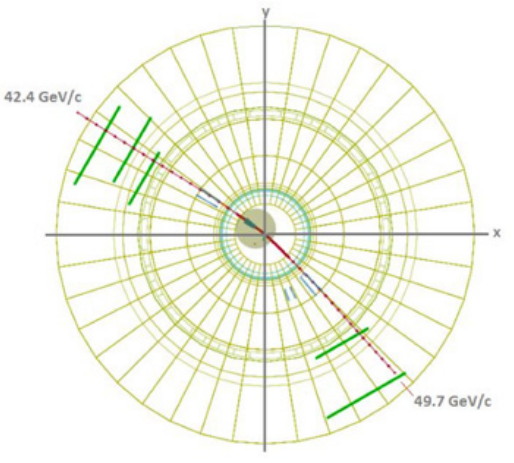

Figure 1: Students use momentum conservation, energy conservation and two-dimensional vector addition to calculate the mass of the $Z$ boson. They gather data from event displays of candidate $Z$ decays from the ATLAS and CMS experiments at CERN's Large Hadron Collider (LHC).

Students at level 2 analyse two variables [13][14]; they calculate descriptive statistics, seek patterns, identify outliers and confounding variables and perform calculations to reach findings; they may also create graphical representations of the data.

Levels 1 and 2 can help prepare students for a masterclass, at the next level up, which uses more data ( $\sim 10^{3}$ authentic events), analysed event-by-event, and provides a setting closer to what physicists experience. The introductory level provides limited amounts of data in short activities to convey key concepts, typically what is done in day-long Masterclasses. With more time, students design their own investigations to access larger data sets in e-Labs [15], which use large data sets analysed in groups with more flexibility and scaffolding to help students understand their tasks. The top level is related to CERN open data portal, which is aimed, not 
exclusively, at university-level skills and challenges. Work has started to address these levels and how we might help students and educators transition from one level to the next.

\section{Basic concepts}

Basics concepts include particle identification (electron, muon, photon, hadron) and event characterisation $\left(\mathrm{W}^{ \pm}, \mathrm{Z}^{0}, \mathrm{H}\right)$. Measuring the ratio of $\mathrm{W}^{+}$and $\mathrm{W}^{-}$occurrences is interpreted in terms of the quark and gluon content of the proton. The invariant mass is successfully mastered (i) to measure properties of various known particles $\left(J / \psi, Y, Z^{0}, K_{s}, D^{0}, \Lambda\right)$, (ii) to discover new ones - Higgs, and (iii) to search for exotic particles and phenomena ( $Z^{\prime} /$ new forces). Other achievements include understanding the concepts of event, statistics and signal significance, on one hand, and simulation, modelling and hypothesis testing, on the other hand.

Powerful and popular educational materials have been developed that introduce the various concepts to the students. Let us concentrate on the invariant mass technique [16][17] [18]. Scalable web-plotting tools, such as OPloT [19], allow submission and automatic combination of all measurements performed. This allows for prompt access to results for further discussion within institutes and during videoconferences held at the end of a Masterclass day. The CMS WZH masterclass measurement uses CIMA [20].

As demonstrated by Figure 2 obtained in one of the 2015 ATLAS $Z$ path days, students identified and measured properties of three known particles, and "discovered" a fourth one, a simulated signal at $1 \mathrm{TeV}$ mixed to the data they analysed. An advanced version of the measurement may feature continuum background as measured in real-life experiments. The main goal, however, was to convey a powerful technique used to measure and identify shortlived particles and to even discover new ones.

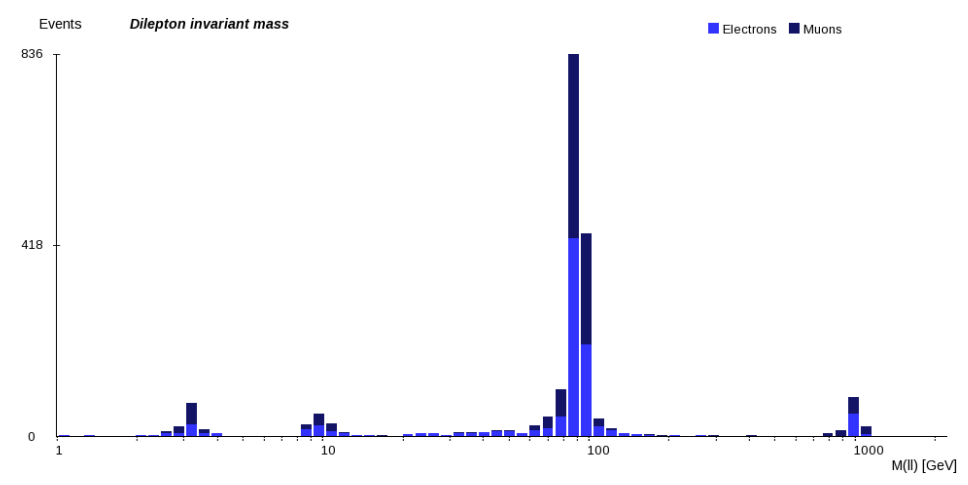

Figure 2: Invariant mass of $e^{+} e^{-}$and $\mu^{+} \mu^{-}$pairs showing known particles as bumps at 3 $\mathrm{GeV}(\mathrm{J} / \psi), 10 \mathrm{GeV}(\mathrm{Y})$ and 90 $\mathrm{GeV}\left(Z^{0}\right)$ all based on real ATLAS data. A signal of a 1 TeV hypothetical Z' based on simulated data is mixed in the data sample analysed by the students.

The lack of statistics and the high background level complicated the measurement related to the Higgs discovery data [18]. The $2 \mathrm{fb}^{-1}$ of ATLAS data made available were not enough for the students to fully enjoy the clear signals obtained in the invariant mass of di-photons and four-leptons and published by ATLAS and CMS. With the help of simulated events, the concept of signal significance was clearly made to the students, see details in Figure 3.

With the advent of LHC Run 2 at $13 \mathrm{TeV}$, new discoveries may be made. It is our intention to prepare for such exciting moments. The main goal of this article is to make use of simulations of some of the popular new physics scenarios and with the help of simple concepts to convey such discoveries to students if and when made. We have already introduced a new 
particle, the Z', a hypothetical heavy partner of the Z boson. It is predicted by some theories beyond the Standard Model that require the introduction of a new weak force. This gave the students the possibility of really discovering something new and unexpected, and allowed them to see how a new particle could be discovered at the LHC.
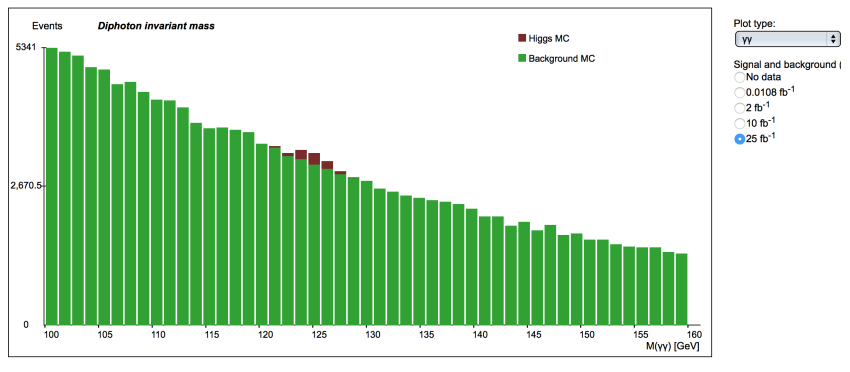

Figure 3: Di-photon invariant mass showing a Higgs signal on top of an abundant SM background. Simulation corresponding to the entire data set recorded by ATLAS (25 $\left.f b^{-1}\right)$. Although the data made available to the students is a factor of 10 smaller. By comparing the corresponding plots when raising and lowering the luminosity the students learn about signal significance.

\section{Basic concepts to follow LHC 'heartbeats' and influence textbooks and teaching methods}

Two of the greatest mysteries in physics today are the nature of dark matter (DM) and the behaviour of the gravitational force at a subatomic level. Why is gravity much weaker than Nature's other fundamental forces? Are there more space dimensions than the usual known 3 , which would allow gravity-related phenomena to occur at the LHC energy era?

More concepts must be brought in if we want to introduce and convey new phenomena, such as dark matter, dark energy, extra space dimensions, graviton resonances, etc. There is an urgent need to extend the educational materials to follow LHC 'heartbeats' to share any new future discovery and influence textbooks and teaching methods. Interesting discussions have started within IPPOG on how to introduce more concepts, should new phenomena be discovered at the LHC. The material presented here can and will be adapted to the various levels discussed in Section 2. In particular, $Z$ path options prepared for future masterclasses are documented below. Research projects based on large LHC data samples open new possibilities for university students and result in a strong support to both the theoretical and experimental lectures. New features are implemented into the $Z$ path, as well as to the ATLAS $W$ path and CMS WZH and Jpsi paths, that will serve both high school and more advanced students. Work is also ongoing on the other LHC measurements. For example the LHCb announcement of pentaquark candidates (bound-states made of 3 quarks and a pair of quark-antiquark) should be explained and shared with students with the help of the invariant mass technique.

Tools to analyse larger datasets also allow more concepts to be brought closer to students. Let us first consider a case of new physics that does not require substantial changes but still needs some introduction and motivation. We start by describing the exploitation of the invariant mass technique to account for additional possible discoveries. We continue concentrating on the $Z$ path.

\subsection{Invariant mass and Gravity}

A spectrum of graviton excitations is present in some theoretical models that include extra space dimensions. Narrow graviton resonances can be detected in various final states. 


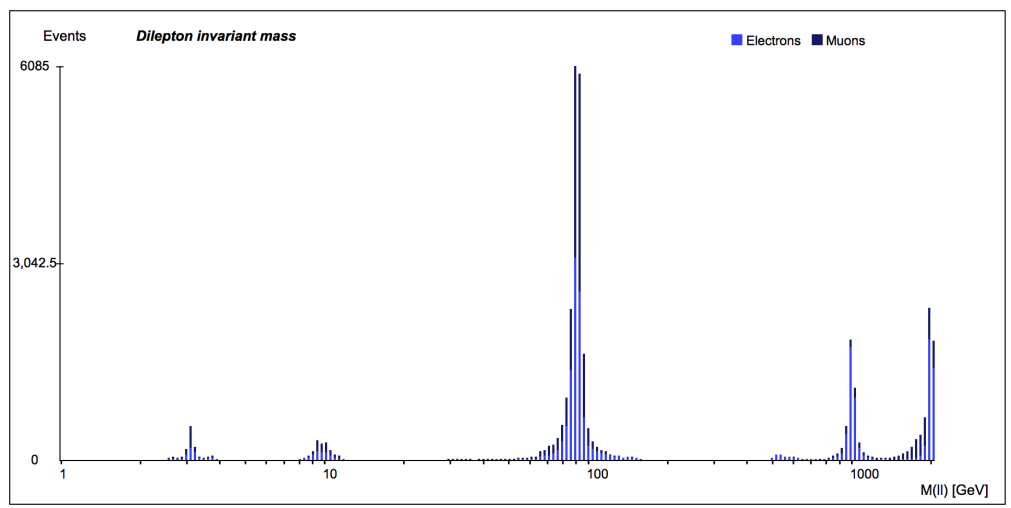

Figure 4: Invariant mass of di-leptons. In addition to the bumps shown in Figure $2\left(J / \psi, Y, Z\right.$ and $\left.Z^{\prime}\right) a$ graviton resonance is added at $2 \mathrm{TeV}$, as well as a Supersymmetry contribution just below the $Z$ mass. The latter is detailed in Figure 6.

In addition to the signals already shown in Figure 2, a simulated graviton $(G)$ resonance is added to the di-lepton invariant mass at $2 \mathrm{TeV}$ (Figure 4). A question immediately arises: how to differentiate the $Z^{\prime}$ and $G$ particles? Modelling of the new physics scenarios (as well as of the Standard Model) is needed here and may be challenging to introduce. Facts, mainly based on properties of the studied objects, such as coupling to final state particles, can be presented to the students. Here is a possibility: the di-lepton distribution shows both the new gauge boson $Z^{\prime}$ and graviton $G$ resonances, but not the Higgs, because the latter has very weak coupling to light leptons (electron, muon). Students have learned in the past that the Higgs boson shows up in both di-photon and di-Z-boson (4 leptons) final states. What about the Graviton? It couples to energy and momentum and a resonance would show up in di-photons (Figure 5 (bottom, right)) and di-bosons (available but not shown here), as well as di-leptons (Figure 5 (top, right)).

Spin or "intrinsic" angular momentum is a property that differentiate various particle types. Although the particles we discussed above are all bosons, meaning they have integer spin quantum numbers, the Higgs has spin 0 , the $Z^{\prime}$ and $Z$ spin 1 , and the Graviton spin 2. Knowing the spin is a way to further characterise a newly observed resonance. This can be done by studying the angular distribution of the decay products in the reference frame of the decaying particle. A spin-0 particle would lead to an isotropic distribution. The higher the spin, the more complicated would be the pattern. Advanced enough students would take advantage and learn a lot from such a measurement.

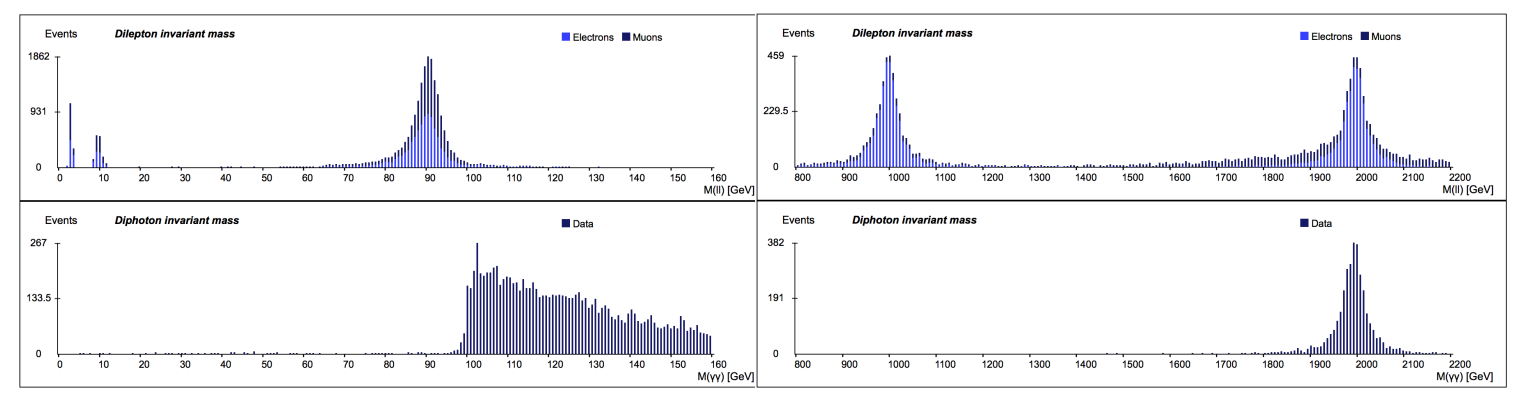

Figure 5: The invariant mass distribution for di-leptons (top, shown in Figure 2) features the real data (J/ $\psi$, Y and Z) (left), a simulated $1 \mathrm{TeV} Z^{\prime}$ and a simulated $2 \mathrm{TeV}$ graviton resonance (right). The diphoton distribution (bottom) shows no Z, but the Higgs data (left, a $100 \mathrm{GeV}$ selection cut was applied). A $2 \mathrm{TeV}$ graviton is visible, but no Z' at $1 \mathrm{TeV}$ as the $Z^{\prime}$ (and Z) cannot decay into di-photons (right). 


\subsection{Missing transverse energy, Dark Matter and Supersymmetry}

Students who performed the ATLAS $W$ and CMS WZH path masterclasses were introduced to the concept of missing transverse energy. They associated it in the display programs they used as the undetected neutrino. The $W$ particles they identified are merely a charged lepton (electron or muon) and an undetected neutrino in the form of missing momentum displayed as a red or yellow dotted line (Figure 7). A signature of the $\mathrm{W}$ boson in a mass plot would need the introduction of a new variable. Transverse mass and "Jacobian peak" must now follow the already mastered invariant mass and "Gaussian peak". Measurements that use the $W$ should accommodate a search for new charged W' bosons, which are often predicted together with the $Z^{\prime}$ already at work in the $Z$ path.

The neutrino is widely accepted, although it is a very light "dark matter" in most HEP experiments. It is "identified" in large detectors the way we would like to detect DM directly through a recoil of some matter particle. Neutrinos are too light to entirely explain the DM "problem" but constitute "background" when searching for DM. Particle physics solutions to DM must involve new particles expected often to be Weakly Interacting Massive Particles (WIMPs).

Supersymmetry (SUSY) is a Standard Model extension, which proposes to describe integer spin particles (bosons: force particles ( $\gamma, \mathrm{W}, \mathrm{Z}$, gluon) and Higgs) and half-integer spin particles (fermions: leptons and quarks) in one theoretical framework, thus doubling the number of fundamental particles. SUSY predicts 5 Higgs bosons, 4 of which are searched for at the LHC. SUSY features the Lightest SUSY Particle (LSP) as a possible DM candidate.

Figure 6 contains a SUSY contribution from final states with di-leptons. No resonance shows up in this case because some information is missing. We considered a search for SUSY in di-lepton final states stemming from decays of gauginos (superpartners of gauge bosons) or sleptons (superpartners of leptons) and where the missing energy is carried by the neutralino, which is the LSP and DM candidate. The signature in Figure 6 gives is an endpoint edge in the di-lepton invariant mass distribution at $\sim 100 \mathrm{GeV}$. The simplified model used predicts a mass of $99.78 \mathrm{GeV}$. The challenge to reconstruct the end-point and have access to some SUSY particle masses will be challenging when Standard Model background processes are added.

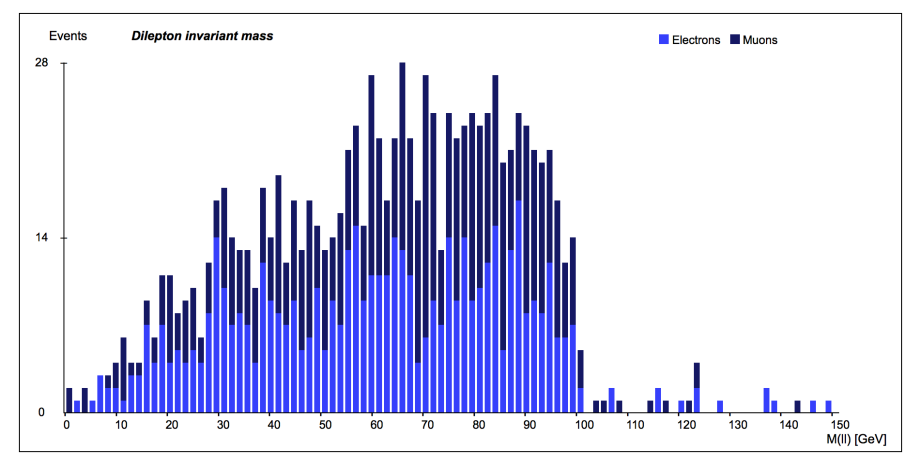

Figure 6: Invariant mass of dileptons obtained with a simulated SUSY signal leading to a final state with two leptons (simplified model). The figure features a wide distribution with a clear end-point edge at $\sim 100$ $\mathrm{GeV}$.

The missing transverse energy is shown as a function of the invariant mass in Figure 8 for SM background (black) and another more general SUSY model (red). For values of ETmiss larger than $100 \mathrm{GeV}$ the SUSY signal is drastically enhanced (Figure 9). 


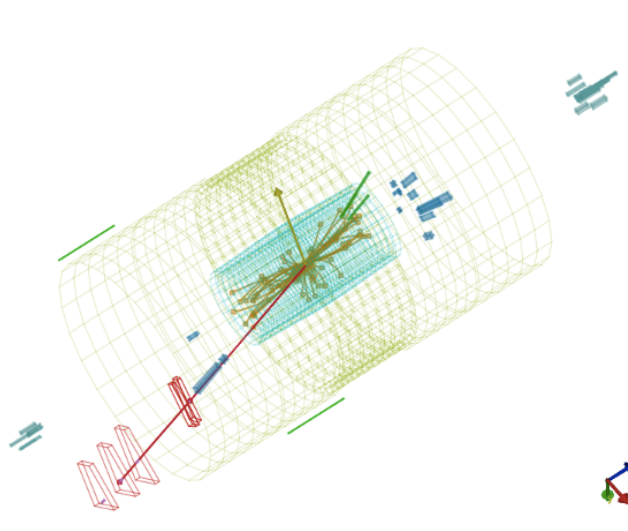

Figure 7: CMS event display showing $a W$ boson candidate from the CMS WZH masterclass measurement. Students use ETmiss (green/yellow up-going arrow) to help identify the parent particle. The $W$ boson candidate decays to a muon (red line) and a neutrino. The Neutrino momentum is inferred from the measured muon and hadrons (tracks shown as green lines and energy deposits in blue).

Whenever new concepts and background material are introduced it is important to be careful with the level and the amount of new information and find out how far can't we go? Which basic concepts must be taught to prepare for new discoveries and how? How to introduce related concepts into "textbooks" and/or educational materials? Whereas it is difficult to explain and convey the hierarchy problem, it should be possible to convey the way we search for DM.

Should a signal be observed at the LHC a remaining challenge will be to differentiate a DM interpretation from others leading to missing transverse energy. Some signatures (jet or photon + graviton G, where G disappears in extra dimensions and causes large missing energy) are similar for graviton and DM (mono-jets, mono-photons). Direct DM searches will be complementary to direct DM production at the LHC. They will be an essential component in order to link any LHC findings to the large scale astrophysics effects observed at the level of galaxies.

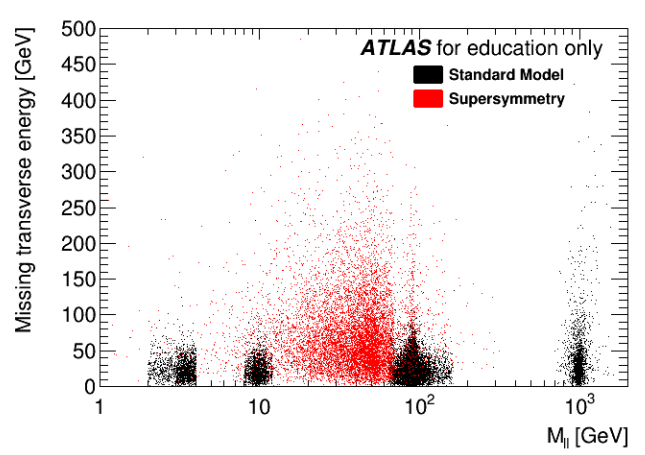

Figure 8: ETmiss vs Mll. A SUSY signal (red) is added to the masterclass samples.

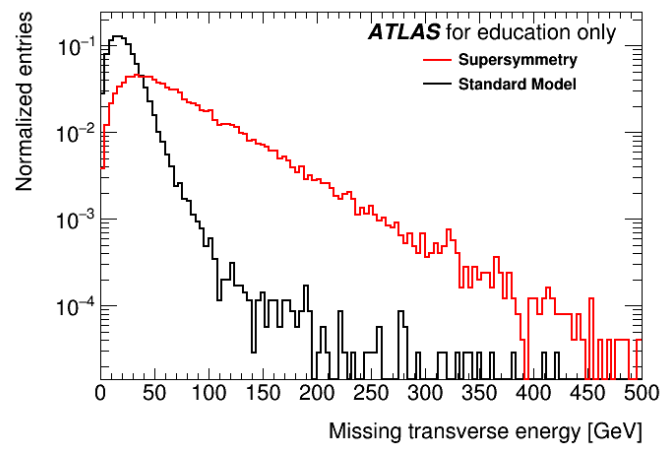

Figure 9: ETmiss for a SUSY signal (red) and the SM masterclass samples (black).

\section{Summary and outlook}

To summarise, we have the ambition to tune and expand the educational material to follow the LHC 'heartbeats' and to influence the textbooks and teaching methods already at the level of high schools. While introducing new concepts and ideas gradually we also want to take advantage of high data statistics to improve the current measurements. Features are already implemented to satisfy high school and more advanced university students. Event-by-event 
visualisation with display programs and batch analysis are complementary. Non-biased and larger data samples will offer the opportunity to perform real analyses, including signal vs background studies and resonance fits, and to directly compare student results to published ones, including theoretical interpretations. In addition to the feeling of working with fresh, real data, larger statistics allow beautiful textbook hands-on activities.

We are looking forward to working with and sharing new real data with students. Let us hope that the LHC Run 2 at $13 \mathrm{TeV}$, which has already started, will come with some surprises and that some of the simulated scenarios shown here will become reality.

\section{References}

[1] M. Bardeen et al., International Masterclasses in the LHC era, CERN Courier, May 2014, http://cerncourier.com/cws/article/cern/57305

[2] LHC@InternationalMasterclasses, http://atlas.physicsmasterclasses.org/en/index.htm

[3] ATLAS W-path- http://atlas.physicsmasterclasses.org/en/wpath.htm

[4] ATLAS Z-path - http://atlas.physicsmasterclasses.org/en/zpath.htm

[5] CMS WZH-path - http://cms.physicsmasterclasses.org/pages/cmswz.html

[6] CMS Jpsi-path - http://cms.physicsmasterclasses.org/pages/cmsjpsi.html

[7] ALICE measurement - www-alice.gsi.de/masterclass/

[8] ALICE ROOT-based - http://aliceinfo.cern.ch/public/MasterCL/MasterClassWebpage.html

[9] LHCb measurement http://hhcb-public.web.cern.ch/lhcb-public/en/LHCb-outreach/masterclasses/en/

[10] TOTEM masterclasses - http://totem-experiment.web.cern.ch/totem-experiment/education/

[11] CERN open data portal - http://opendata.cern.ch

[12] QuarkNet Data Portfolio, https://quarknet.i2u2.org/data-portfolio/

[13] CMS Data Express - https://quarknet.i2u2.org/data-portfolio/activity/cms-data-express

[14] TOTEM Data Express - https://quarknet.i2u2.org/data-portfolio/activity/totem-data-express

[15] CMS eLab - http://www.i2u2.org/elab/cms/home/project.jsp

[16] F. Ould-Saada, Bringing LHC data into the Classroom, PoS ICHEP2012 (2013) 559

[17] M. K. Bugge et al., ATLAS Masterclasses - $W$ and $Z$ path physics and presentation of the $Z$ path measurement, EPJ Web of Conferences 71 (2014)

[18] M. Pedersen et al., Sharing ATLAS data and research with young students, PoS ICHEP2014 (2015)

[19] V. Morisbak et al., OPloT, Oslo Plotting Tools, http://cernmasterclass.uio.no

[20] CIMA, CMS Instrument for Masterclass Analysis - https://www.i2u2.org/elab/cms/cimal 